\title{
Non Destructive TeSTiNg OF FORGE WELDING JOINT ERRORS
}

\section{Zdenka Keran, Morana Mihaljević, Amalija Horvatić Novak \& Biserka Runje}
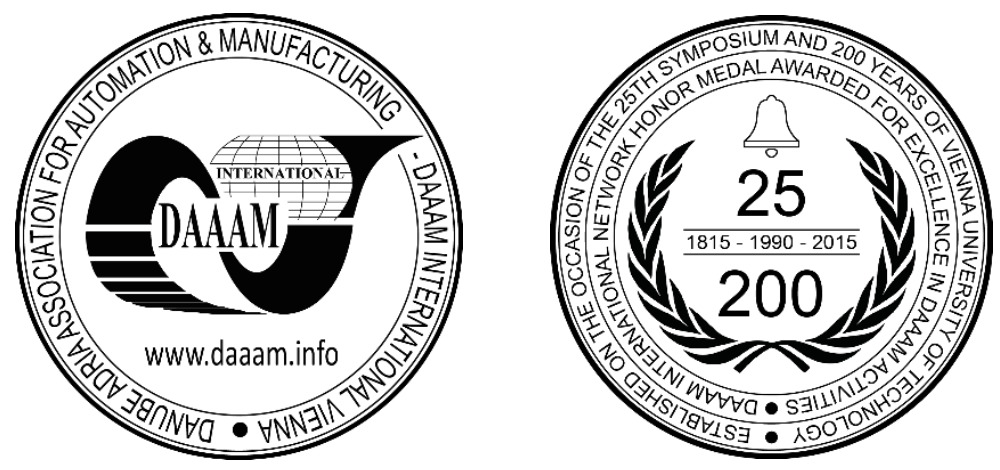

This Publication has to be referred as: Keran, Z[denka]; Mihaljevic, M[orana]; Horvatic Novak, A[malija] \& Runje, B[iserka] (2018). Non Destructive Testing of Forge Welding Joint Errors, Proceedings of the 29th DAAAM International Symposium, pp.0169-0175, B. Katalinic (Ed.), Published by DAAAM International, ISBN 978-3-90273420-4, ISSN 1726-9679, Vienna, Austria

DOI: $10.2507 / 29$ th.daaam.proceedings.024

\begin{abstract}
Forge welding is a solid state process whereby the melting temperature is not reached. Hammer blows cause permanent deformation and assure metallurgical contact between two elements to be welded together. The main characteristic of the weld is that two surfaces are joined together by whole contact area. That is why, in industrial conditions, an error occurrence is very often. For testing and measuring purposes an experimental aluminium sample has been forge welded. An error has been deliberately created in welding process, with previously known dimensions and characteristics. This work compares two possible non destructive testing techniques for such weld type: Computed Tomography - CT and Computer Radiography - CR and gives recommendation for its usage.
\end{abstract}

Keywords: forge welding; non destructive testing; computed tomography; computer radiography

\section{Introduction}

Forge welding is a solid-state welding process that joins two pieces of metal by heating them to a high temperature and then hammering those together [1]. The process is one of the simplest methods of joining metals and has been used since ancient times. Forge welding is versatile, being able to join a host of similar and dissimilar metals. Forge welding between similar materials is caused by solid-state diffusion. This results with a weld that consists of only the welded materials without any filler or bridging materials. The temperature required to forge weld is typically (50 to 90) percent of the melting temperature. The metal may take on a glossy or wet appearance at the welding temperature. Care must be taken to avoid overheating the metal to the point that it gives off sparks from rapid oxidation (burning).

An error occurrence, placed on the weld area, is very often in industrial conditions. Because two large surfaces are placed together, it is almost impossible to avoid an accidental occasional occurrence of impurities, oxidation products or secondary material particles in the weld area, no matter how ideal production conditions are. Those errors, however, can be harmful in occasions when construction is in danger to be weakened.

The idea of this research work is to use two non destructive techniques for inspection of welding area, collect achievable data and give its comparison in order to conclude which one of them would provide better and more complete information about possible welding process errors. 
For described reason such forge welded sample has been produced, with deliberate error, secondary material particle of well known geometry, included on the welded surface area. The sample consists of two aluminium cylindrical parts and steel flat washer between them. Aluminium parts have been heated on $400{ }^{\circ} \mathrm{C}$ and welded together by forging on pneumatic forging hammer. Steel flat washer has been put between welded surfaces before the forging process. Schematic preview of the sample is given on Fig.1.

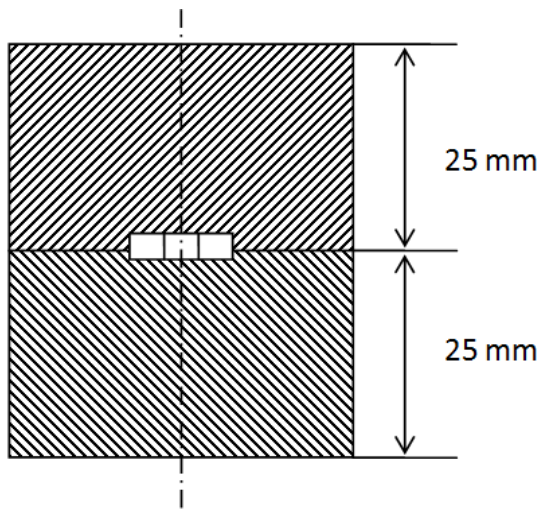

(a)
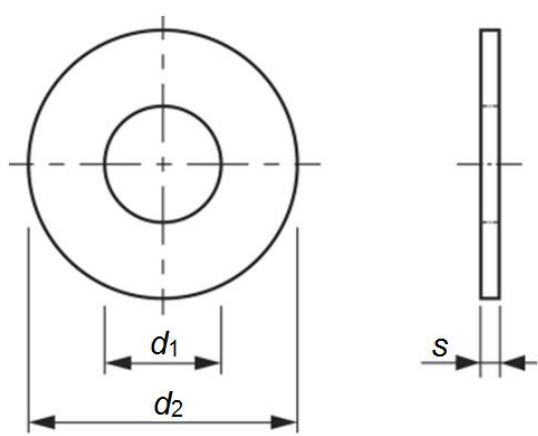

$$
d_{1}=6,4 \mathrm{~mm} ; d_{2}=12 \mathrm{~mm} ; s=1,6 \mathrm{~mm}
$$

(b)

Fig. 1. Schematic preview of sample: a) scheme of experimental welded aluminium sample, b) scheme of flat steel washer.

The welded joint has been examined using two non-destructive techniques Computer Radiography - CR and Computed Tomography - CT. These techniques provide testing over whole surface area and are suitable for larger surfaces than other non-destructive testing methods.

Advantages and disadvantages of $C R$ : In radiographic testing, information is presented pictorially. A permanent record is provided, which can be viewed at a time and place distant from the test. This type of testing is useful for thin sections and is suitable for any material. Sensitivity is declared on each film. Computer radiography is commonly used as non destructive method for inner structure analysis of some components. Its main feature is the possibility of test components throughout the volume regardless of geometric complexity. Since computer radiography imaging plate replaces industrial film, digital images takes less space than film for storage. Also has a greater dynamic range of relative exposure and „under exposed" or „overexposing“ could not occur. Advantages of this technique in regard to radiographic film technique is shorter exposure time up to $80 \%$ of the time required for exposing conventional film and due to greater dynamic range of digital detectors "sandwich technique" is not required. On the other hand, radiography requires skills in choosing angles of exposure, operating equipment, and interpreting indications. It requires safety precautions. Radiography is not suitable for several types of testing situations. For example, radiography is inappropriate for surface defects and for automation, unless the system incorporates fluoroscopy with an image intensifier or other electronic aids. Radiography generally can't cope with thick sections, and the testing itself can pose a possible health hazard. Film processing and viewing facilities are necessary, as is an exposure compound. With this method, the beam needs to be directed accurately for 2D defects. Also, radiographic testing does not indicate the depth of a defect below the surface [2] [3] [4].

Advantages and disadvantages of CT: It is a non-tactile and non-destructive measurement method and its application is widely recommended with problems where geometry and characteristics of inspected part are not accessible with usage of conventional measurement method e.g. tactile measurement methods [5] [6]. It allows us insight into all characteristics of internal geometry as well as their exact position in the object. From the fact that it is a non-tactile method, there is no fear of damaging the object during scanning process and from the same reason, it is method suitable for measurement of deformable objects [7]. One more advantage, which can be in some situation really useful is that by using CT it is possible to inspect parts in assembled stated without disassembled them. However, it is a measurement method where metrological traceability is not achieved and measurement uncertainty is not evaluated [8] [9]. It is a method with no defined standard, so measurement approach and selection of scanning parameters rely on operator skills and earlier experiences. Also, scanning process can last up to several hours, but once the part is scanned and model generated, all information about part are accessible from same model.

\section{Testing by computed radiography}

Over the last decade there has been an intensive development of computer radiography and at the same time there is an acceptance of technology in the field of industrial radiography. Computer radiography systems use a photostimulating panel which during exposure partially converts X-radiation into the light, and partially saves it as latent picture. Afterwards this latent picture is converted into the digital record with usage of scanner imaging plate. 
Observed part, forge welded aluminium with deliberate error, and was inspected with usage of computed radiography method. The elements were filmed on imaging plate with X-ray and processed with computer radiography software Starrwiev VMI 5100MS.

\begin{tabular}{|l|c|}
\hline \multicolumn{1}{|c|}{ Input parameter } & Amount \\
\hline Voltage, $\mathrm{kV}$ & 125 \\
\hline Current, $\mathrm{mA}$ & 4 \\
\hline Source to film distance, $\mathrm{mm}$ & 1000 \\
\hline Imaging plate & Blue $\mathrm{XL}$ \\
\hline X-ray spot size, $\mathrm{mm}$ & 2 \\
\hline
\end{tabular}

Table 1. Setups for CR scanning

After the scanning process, imaging was processed in software ISee.

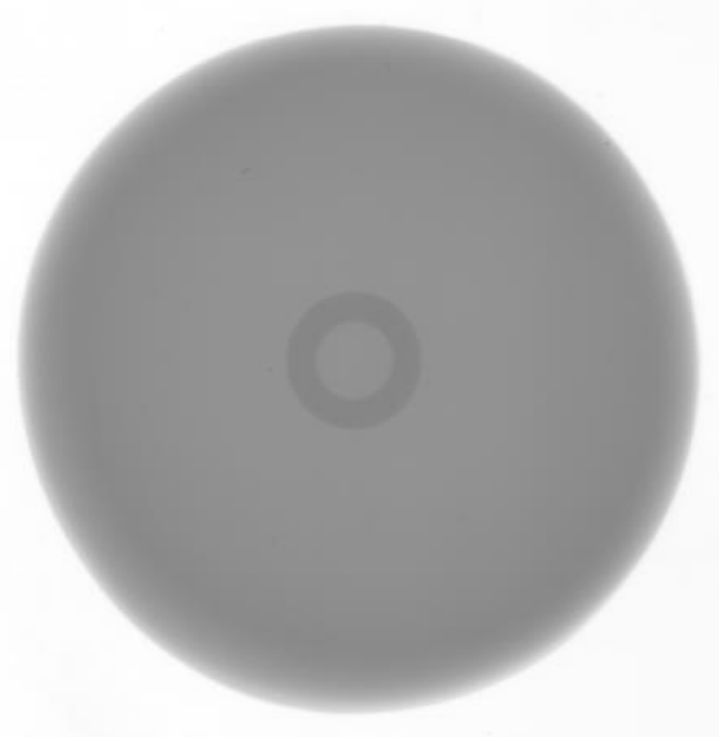

Fig. 2. Elements filmed with CR

Measured dimensions were inside and outside diameter of perceived object. Measurement results are given in Figure 3. and Table 2 as arithmetic mean of three repeated measurements.
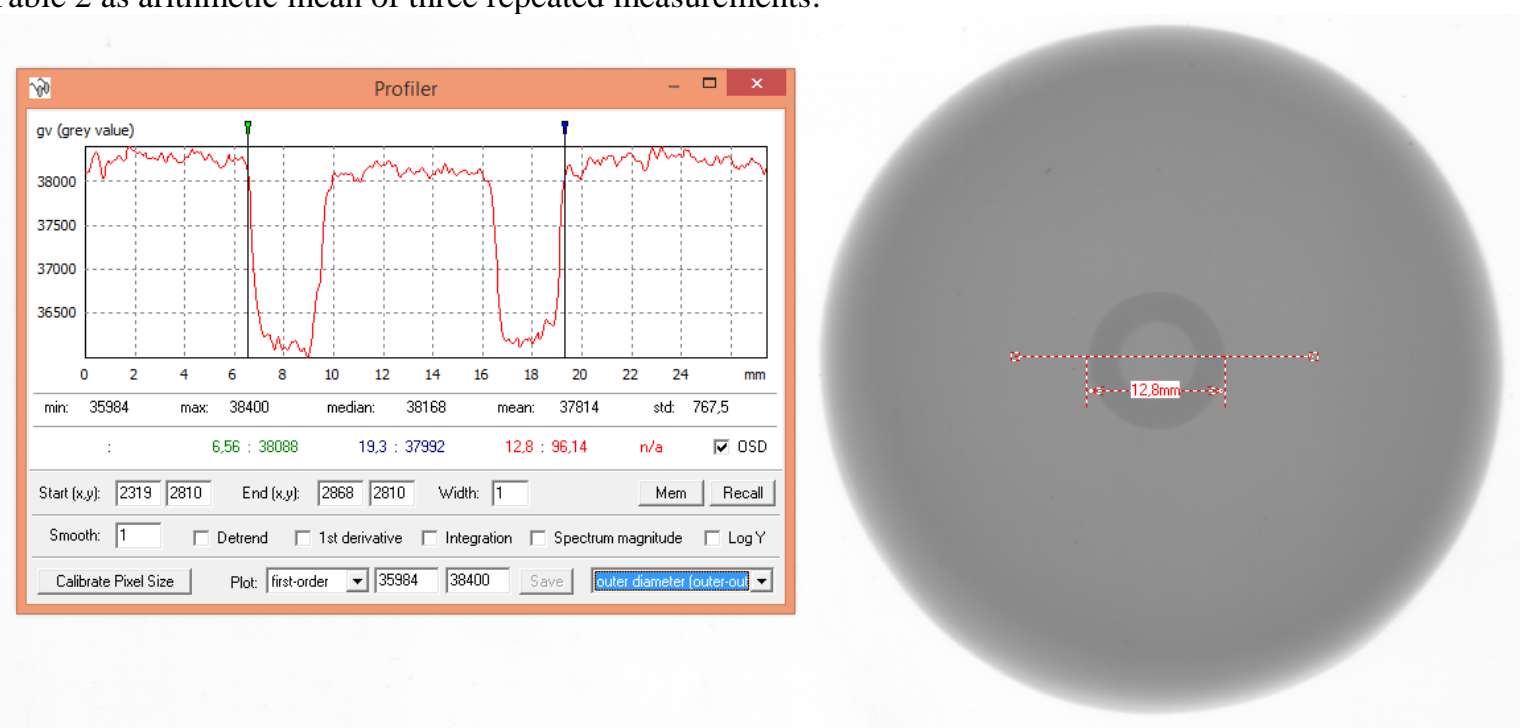

Fig. 3. CR measurement of the outer diameter of observed object 

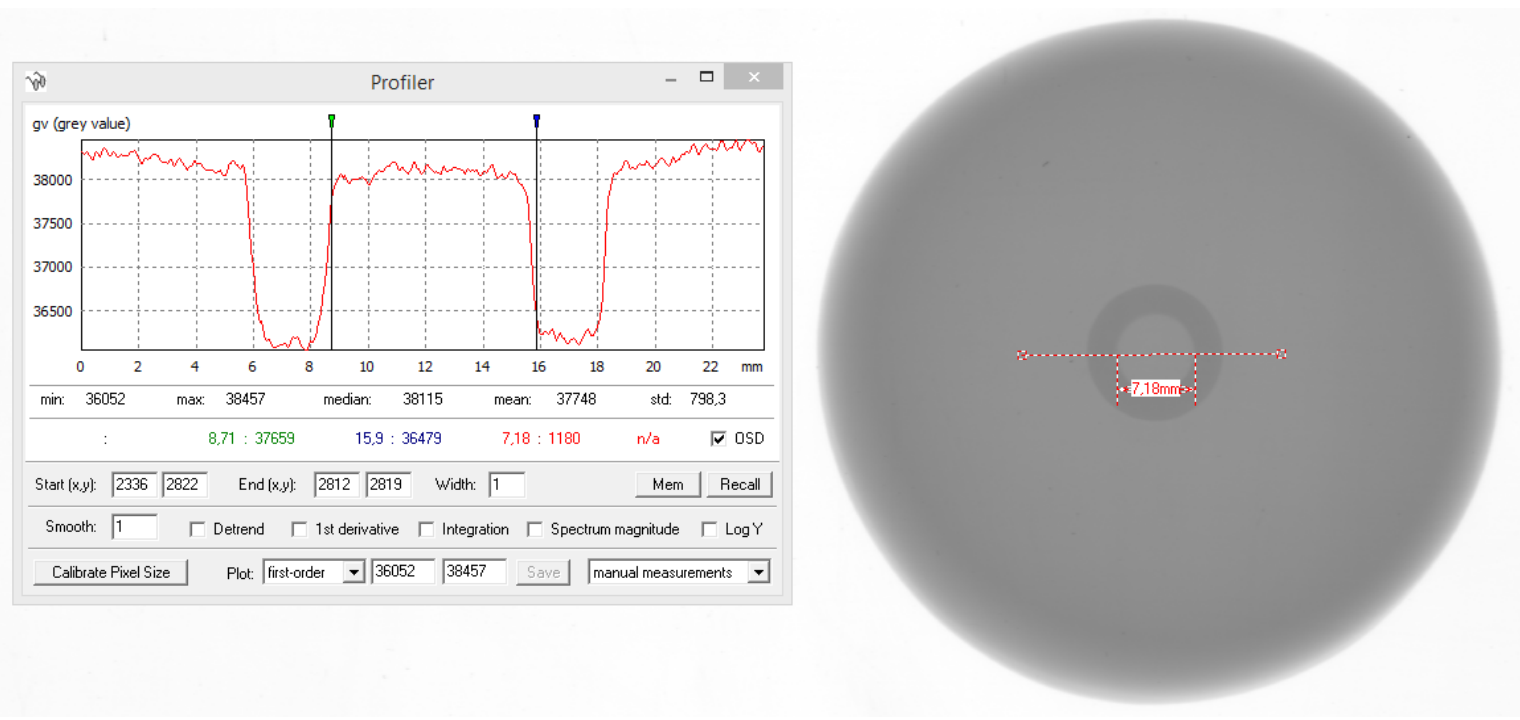

Fig. 4. CR measurement of the inner diameter of observed object

\begin{tabular}{|l|c|c|}
\hline Measured characteristic & Measurement result & $\boldsymbol{U}, \boldsymbol{k}=\mathbf{2 , \boldsymbol { P } = \mathbf { 9 5 } \%}$ \\
\hline Outer diameter & $12,70 \mathrm{~mm}$ & $0,230 \mathrm{~mm}$ \\
\hline Inner diameter & $7,15 \mathrm{~mm}$ & $0,180 \mathrm{~mm}$ \\
\hline
\end{tabular}

Table 2. CR measurement results of inner and outer diameter of observed object

Because of lower geometric sharpness, the outer diameter shows larger deviations than the inner one.

\section{Testing by computed tomography}

Computed tomography - CT is a method that enables insight into internal geometries and structures of inspected object without need for its destruction. It is a method used in a medicine and in material analysis for over 30 years, and for the last 15 years it is also used in field of dimensional measurements. Taking into account nowadays trends in production, based on additive technologies, non destructive analysis and measurements methods becomes necessary in industries. Considering the fact that one of the main advantages of the method is possibility to conduct different analysis on the 3D model e.g. pores or inclusion analysis and dimensional measurements of different features, CT measurements seem like reasonable choice.

The process of testing by CT can be subdivided into three sub processes: CT scanning, reconstruction process and conduction of analysis. Scanning process is carried out in the CT scanner where X-ray source generates X-radiation which is through the focus emitted on the object under investigation. Object is placed on the rotational table while detector captures 2D images of scanned part (Fig. 5).

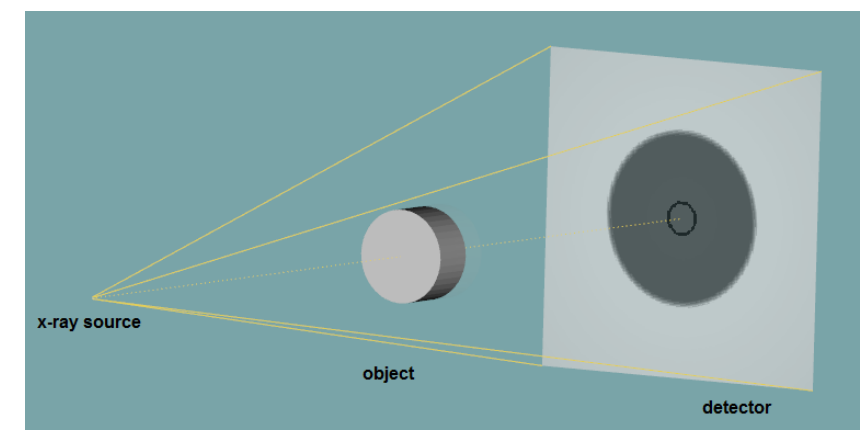

Fig. 5. Principle of CT scanning

Next step includes reconstruction process from large number of captured 2D images where 3D voxel model is made. Finally, measurement analysis is conducted on the model, either with simple geometry fitting approach or by comparing voxel model and CAD model, i.e. nominal and actual geometry. 
Experimental forge welded part, was inspected with usage of computed tomography method. Scanning process was carried out in CT device Nikon, model XT H 225 under conditions presented in the Table 3.

\begin{tabular}{|l|c|}
\hline Input parameter & Amount \\
\hline Voltage, $\mathrm{kV}$ & 220 \\
\hline Current, $\mu \mathrm{A}$ & 180 \\
\hline Cooper filter, mm & 3 \\
\hline Geometrical magnification & 3,94 \\
\hline Number of projections & 720 \\
\hline
\end{tabular}

Table 3. Setups for CT scanning

After the scanning process, 3D model was generated with usage 3D CT Pro software, while analysis and measurements were later conducted on the obtained 3D model with usage of VolumeGraphics Studio Max 2.2 software.

Already in first step of whole process, setting the parameters of the CT scanning process, for the first time, inclusion into the object was observed (Fig. 6). Somewhere in the middle of the aluminium object was observed presence of the circle object. From the difference in gray value it can be stated that this inclusion is made from material with higher density compared with aluminium density.

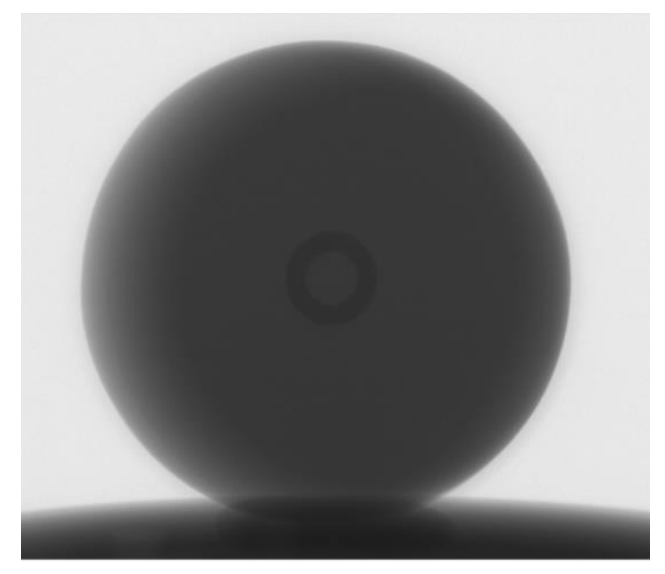

Fig. 6. 2D image obtained during CT scan

After the model was generated, dimensional measurement of identified inclusion was conducted. Measured dimensions were inside and outside diameter of perceived object. Chosen measurement approach involved fitting simple geometry objects, in this case, cylinders, using Gaussian mathematical approach (Fig. 7). Number of points used to fit simple objects was set to 1000 .

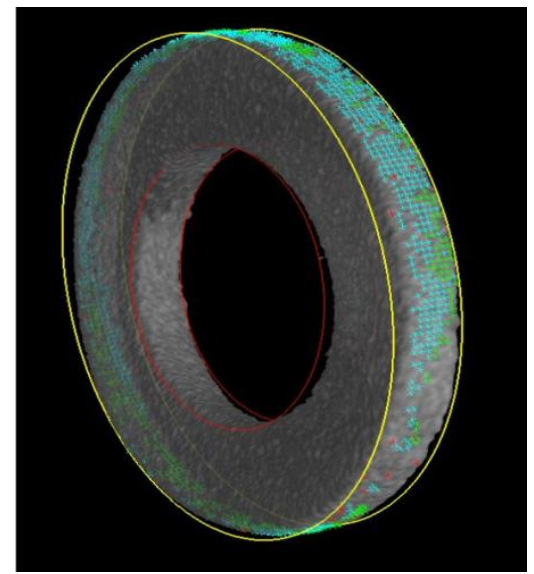

a)

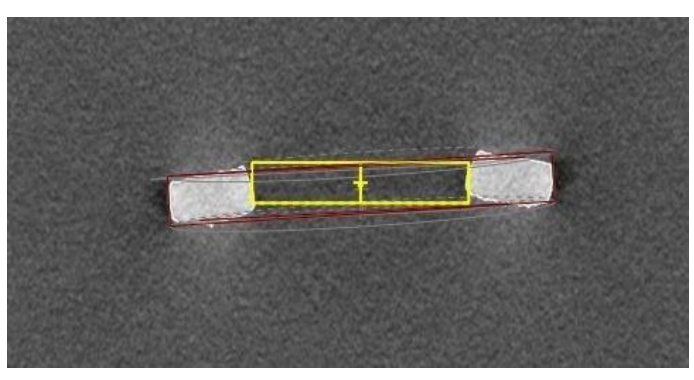

b)

Fig. 7. Measurement approach - fitting simple objects: cylinder a) outer diameter, b) inner diameter 
Gaussian approach minimizes the sum over all deviations of all fit points relative to the fitted reference object. For a large number of fit points the distribution of the deviations will usually result in a Gaussian shape. The reference object is positioned in such a way that the sum of negative deviations equals the sum of positive deviations [10]. Scaling correction of obtained data sets was performed using calibration rod with two ruby spheres. Distance between sphere centres was used to correct nominal voxel size. In total three repeated measurements of the sample were conducted. Measurement results are expressed as arithmetic mean of obtained results and are given in Table 4.

\begin{tabular}{|c|c|c|}
\hline Measured characteristic & Measurement result & $\boldsymbol{U}, \boldsymbol{k}=\mathbf{2 ,} \boldsymbol{P}=\mathbf{9 5} \%$ \\
\hline Outer diameter & $12,59 \mathrm{~mm}$ & $0,090 \mathrm{~mm}$ \\
\hline Inner diameter & $7,08 \mathrm{~mm}$ & $0,070 \mathrm{~mm}$ \\
\hline
\end{tabular}

Table 4. Measurement results obtained with CT

\section{Comparison of the results}

Both outer and inner diameters were measured using two non-destructive measurement methods: computed radiography and computed tomography. Results are presented graphically in Fig. 8. with given expanded uncertainty $U$, coverage factor $k=2$ and probability $P=95 \%$.
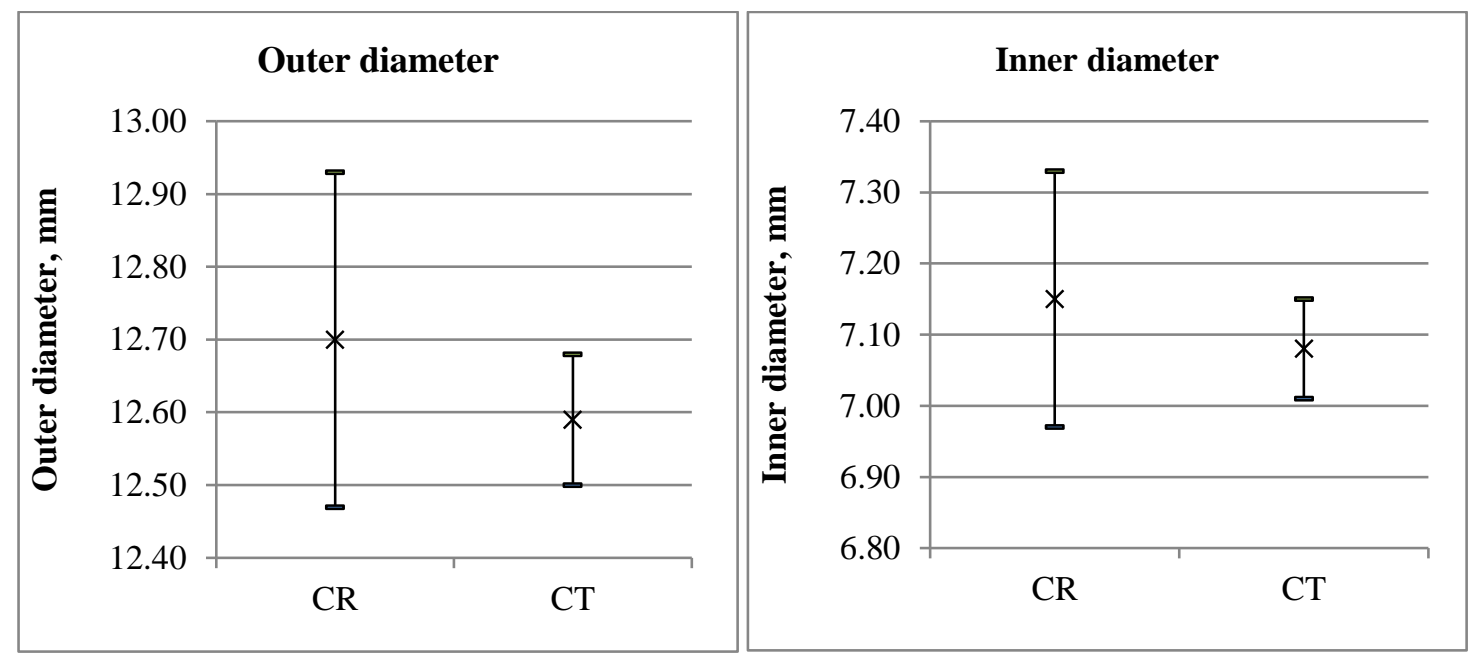

Fig. 8. Measured results with given expanded measurement uncertainty: a) outer diameter, b) inner diameter

In order to validate obtained results, the comparison between measured results was achieved by calculating the En value for the inner and outer diameter.

\begin{tabular}{|c|c|}
\hline Measured characteristic & En \\
\hline Outer diameter & 0,4 \\
\hline Inner diameter & 0,3 \\
\hline
\end{tabular}

Table 5. En numbers

In both cases the En value was smaller than 1 (Table 5), which means that there is a good agreement between results obtained by computed radiography and computed tomography.

\section{Conclusion}

The intention of the research work was to investigate two non destructive methods for inspection of welding area, and give its comparison in order to conclude which one of them would provide better and more complete information about possible welding process errors.

Both methods provide obtaining a blur image of the error generated at the welding area. In doing so, the CR method enables faster achievement of a two-dimensional picture display of the obtained results. The values of measurement uncertainty show that dispersion of the measurement results obtained by the CR method is larger than the dispersion of the measurement results obtained by the CT method. 
Considering that measurement uncertainty is the measure of quality of measurement results, it can be concluded that the CT method yields a better quality of measurement results. The CT method also allows us to obtaine a threedimensional picture display of the error-finding space, however the time it takes to get these results is much longer. In both cases, En values show consistency between measurement results. It can therefore be concluded that both methods can be used in non destructive testing of forge welded joints. Further research in the area should lead to a comparison between many different methods using several criteria.

\section{References}

[1] Nauman, D. (2004). Forge Welding. Controlled Hand Forging. pp. 10-15.

[2] Willcox, M. \& Downes, G. (2006). A review of common nondestructive tests - Assessing each process, its tools, advantages, and disadvantages. Available from: https://www.thefabricator.com/article/testingmeasuring/a-reviewof-common-nondestructive-tests. Accessed: 2018-01-25

[3] Nondestructive Weld Examination. Available from: http://www.lincolnelectric.com/en-us/support/process-andtheory/Pages/nondestructivie-weld-detail.aspx. Accessed: 2018-01-25

[4] Hayes, C. (1998). The ABC's of Nondestructive Weld Examination. Available from: https://www.ndt.net/article/0698/hayes/hayes.htm. Accessed: 2018-01-25

[5] Horvatić Novak, A., Runje, B. \& Stepanić, J. (2017). " Capabilities of industrial computed tomography in the field of dimensional measurements", Advances in Production Engineering \& Management, Vol.12, No. 3, pp.245-253, ISSN: 1854-6250, DOI: 10.14743/apem2017.3.255

[6] Horvatić Novak, A., Runje, B. \& Butković, D. (2016). Influence of geometrical magnification on computed tomography dimensional metrology, Proceedings of the 27th DAAAM International Symposium, pp.0615-0622, B. Katalinic (Ed.), Published by DAAAM International, ISBN 978-3-902734- 08-2, ISSN 1726-9679, Vienna, Austria DOI: 10.2507/27th.daaam.proceedings.090

[7] Welkenhuyzen, F., Kiekens, K., Pierlet, M., Dewulf, W., Bleys, P., Kruth, J.P. \& Voet, A. (2009). Industrial Computer Tomography for Dimensional Metrology: Overview of Influence Factors and Improvement Strategies, Proceedings of the 4th International Conference on Optical Measurement Techniques for Structures and Systems: OPTIMESS 2009

[8] Kruth, J.P., Bartscher, M., Carmignato, S., Schmitt, R., De Chiffre, L. \& Weckenmann, A. (2011). "Computed tomography for dimensional metrology", CIRP Annals - Manufacturing Technology, Vol. 60, No.2, 2011, pp. 821-842, ISSN: 0007-8506, DOI: https://doi.org/10.1016/j.cirp.2011.05.006

[9] Carmignato, S., Pierobon, A., Rampazzo, P., Parisatto, M. \& Savio, E. (2012). CT for Industrial Metrology Accuracy and Structural Resolution of CT Dimensional Measurements, Proceedings of 4th Conference on Industrial Computed Tomography, 2012, Wels, Austria

[10] VGStudio MAX 2.2 - Reference manual, Volume Graphics, Heidelberg, Germany, 2013 